\title{
Analysis of nanoindentation of soft materials with an atomic force microscope
}

\author{
Jacob Notbohm, Benny Poon, and Guruswami Ravichandran ${ }^{\text {a) }}$ \\ Division of Engineering and Applied Science, California Institute of Technology, Pasadena, California 91125
}

(Received 4 May 2011; accepted 2 August 2011)

\begin{abstract}
Nanoindentation is a popular experimental technique for characterization of the mechanical properties of soft and biological materials. With its force resolution of tens of pico-Newtons, the atomic force microscope (AFM) is well-suited for performing indentation experiments on soft materials. However, nonlinear contact and adhesion complicate such experiments. This paper critically examines the application of the Johnson-Kendall-Roberts (JKR) adhesion model to nanoindentation data collected with an AFM. The use of a nonlinear least-square error-fitting algorithm to calculate reduced modulus from the nanoindentation data using the JKR model is discussed. It is found that the JKR model fits the data during loading but does not fit the data during unloading. A fracture stability analysis shows that the JKR model does not fit the data collected during unloading because of the increased stability provided by the AFM cantilever.
\end{abstract}

\section{INTRODUCTION}

In recent years, the importance of the mechanical characterization of biological materials has become evident in the field of mechanobiology, ranging from the diagnosis of diseases like cancer ${ }^{1}$ and malaria ${ }^{2}$ to the understanding of mechanisms in cell biology such as cell growth ${ }^{3}$ and locomotion. ${ }^{4,5}$ Biological materials are commonly soft and inhomogeneous in nature, which can make them difficult to characterize using traditional mechanical experiments. Additionally, biological specimens such as thin protein films and cells often have small sizes or volumes. When mechanical properties of such biological specimens are of interest, nanoindentation provides a means to characterize the mechanical behavior of these specimens. Several studies have been performed detailing the use of a commercial nanoindenter to mechanically characterize biological specimens such as skin $^{6}$ and cartilage. ${ }^{7}$

An alternative instrument to the nanoindenter is the atomic force microscope (AFM), and a nanoindentation experiment with an AFM is shown in Fig. 1(a). The AFM offers several advantages over the nanoindenter: it is a more common instrument; use of different types of cantilevers gives the AFM more flexibility in indenter shape, size, and surface functionalization; and most commercial AFMs come with a fluid cell for experiments with hydrated specimens. Possibly the biggest advantage of the AFM is that unlike a nanoindenter, the AFM does not require a load cell to measure the indentation load. Instead, the indentation force is measured in an AFM by multiply-

\footnotetext{
a)Address all correspondence to this author.

e-mail: ravi@caltech.edu.

DOI: $10.1557 / j m r .2011 .252$
}

ing the measured deflection of the cantilever with the cantilever's stiffness. Compared to the nanoindenter, which has a resolution of approximately $100 \mathrm{nN}$, the AFM can measure forces in the range of pico-Newtons to hundreds of micro-Newtons by simply changing the stiffness of the cantilever. The superior force resolution of the AFM makes it particularly suitable for nanoindentation of soft biological materials, including cells. ${ }^{8}$

Despite these advantages of the AFM, several difficulties have prevented the AFM from gaining widespread use for nanoindentation experiments on soft materials. Because the indentation load is measured by the deflection of the AFM cantilever, the spring stiffness of the cantilever must be accurately known. Fortunately, methods for determining the spring stiffness of an AFM cantilever such as the thermal tune method ${ }^{9}$ are now incorporated into the software of modern AFMs. Additionally, an unknown tip geometry and size can require using electron microscopy $^{10}$ or blind tip deconvolution ${ }^{11}$ to properly compute the elastic properties of soft materials. This problem can be solved by attaching spherical particles of known radius to the AFM cantilevers.

Compared with the solutions to some problems associated with using the AFM for nanoindentation, the effects of adhesion introduce more difficult challenges when indenting soft materials. In addition to surface forces, longer range attractive forces such as van der Waals and electrostatic forces can mask the onset of contact between the AFM probe and the specimen, which creates uncertainty in the location of the contact point. Several studies have shown that contact point uncertainty can cause significant error in the calculation of the specimen's modulus on both soft ${ }^{12-15}$ and hard ${ }^{16,17}$ materials, and these studies have accounted for this uncertainty by shifting the 


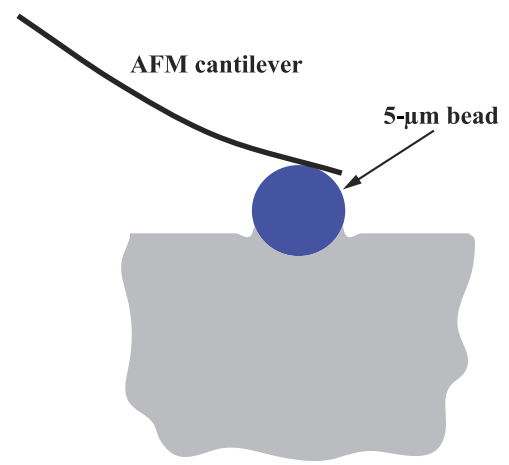

(a)

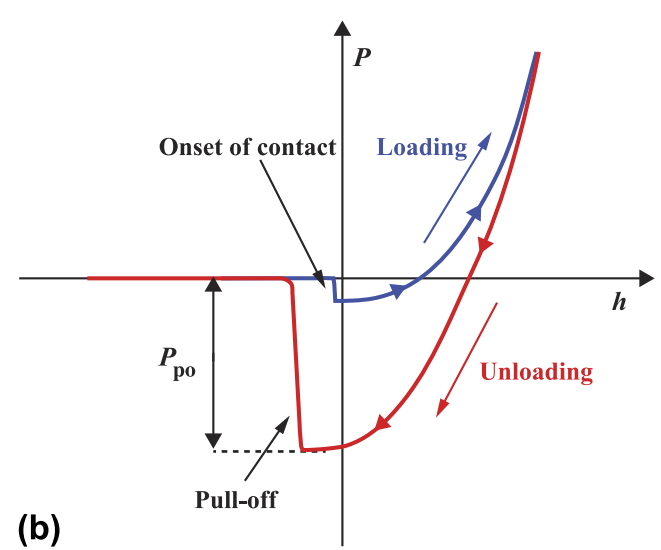

(b)

FIG. 1. (a) Schematic of the nanoindentation experiment with an atomic force microscope (AFM). The polydimethylsiloxane (PDMS) sample deforms as the AFM scanner moves it upward toward the 5- $\mu \mathrm{m}$ glass bead attached to the AFM cantilever. (b) Hypothetical force-indentation curve showing the loading and unloading phases. During loading, the AFM cantilever snaps in to contact with the specimen. During unloading, the cantilever snaps out of contact at the pull-off force, $P_{\mathrm{po}}$.

measured indentation depth data by some constant. However, no standardized method of shifting the data has been adopted. Additionally, the energy release rate changes during a macroscale adhesion experiment on soft materials, ${ }^{18,19}$ so it is unlikely that the energy release rate stays constant during AFM nanoindentation. Furthermore, it has been shown that energy release rate can be dependent on rate. ${ }^{20}$ Because of the nonlinear geometry involved in the contact of the AFM tip to a flat surface, it is unlikely that the rate of growth of contact remains constant during the experiment, which could introduce fluctuations in the energy release rate. As a result of some of these difficulties, many previous nanoindentation studies on soft materials have ignored the effects of adhesion, assuming them to be minimal. ${ }^{12,15}$

The objective of this paper is to properly account for adhesion in a nanoindentation experiment with an AFM on soft materials by applying the well-known JohnsonKendall-Roberts (JKR) model. ${ }^{21}$ The reduced modulus $E^{*}$ of the material is computed and compared with the modulus measured using a uniaxial tension experiment. The methodology is illustrated through experiments using a spherical indenter mounted on an AFM cantilever and a polydimethylsiloxane (PDMS) specimen. The JKR model's assumption of a constant work of adhesion is then discussed in relation to data collected during nanoindentation with AFM. Finally, a fracture stability analysis is employed to explain the reason for a mismatch between the JKR model and the data collected during the unloading phase of the nanoindentation experiments.

\section{THEORY}

The problem of contact between two linear, elastic spheres was initially studied by Hertz, who assumed no surface or shearing forces and small displacements. ${ }^{22}$ This theory can be extended to a spherical indenter and a linear, elastic half space. The analysis shows that for a contact radius $a$ that is much smaller than the indenter radius $R$, the indentation depth $h$ is given by

$$
h=\frac{a^{2}}{R} .
$$

The indentation force $P$ can be shown to be related to the contact radius by the equation

$$
P=\frac{4 a^{3} E^{*}}{3 R}
$$

where $E^{*}$ is the reduced modulus of the specimen, which is given by $E^{*}=E /\left(1-v^{2}\right)$ when the indenter's modulus is much greater than the specimen's modulus with $E$ equal to Young's modulus and $v$ equal to Poisson's ratio.

For the adhesion of soft materials, the famous JKR theory, which considers attractive forces within the contact area caused by surface energy, ${ }^{21}$ can be combined with the Hertz model. The surface energy associated with adhesion has the effect of increasing the radius of contact and the indentation depth. Applying the JKR model gives a new equation for the indentation depth:

$$
h=\frac{a^{2}}{3 R}+\frac{P}{2 a E^{*}} .
$$

One can show that the energy release rate $G$ is given by ${ }^{23}$

$$
G=\frac{\left(\frac{4 E^{*} a^{3}}{3 R}-P\right)^{2}}{8 \pi E^{*} a^{3}}
$$

For systems in equilibrium, the energy release rate $G$ is equal to the work of adhesion $w$. One can show that the contact radius is related to the applied load by ${ }^{23}$ 


$$
a^{3}=\frac{3 R}{4 E^{*}}\left\{P+3 \pi G R+\left[6 \pi G R P+(3 \pi G R)^{2}\right]^{1 / 2}\right\} .
$$

A typical force-indentation curve for a JKR experiment is shown in Fig. 1(b). The force marked $P_{\mathrm{po}}$ on the curve is called the pull-off force, and, in a load-controlled system, it is related to the energy release rate at pull-off $G$ through the equation ${ }^{23}$

$$
P_{\mathrm{po}}=\frac{3}{2} \pi G R
$$

A displacement-controlled experiment is more stable, and pull-off occurs at a smaller contact radius and pulloff force ${ }^{23}$ :

$$
P_{\mathrm{po}}=\frac{5}{6} \pi G R
$$

An often overlooked assumption made when applying the Hertz theory to nanoindentation experiments is that the radius of contact is small. For larger contact radii, the solution of a spherical punch ${ }^{24}$ may be considered as well. Maugis ${ }^{25}$ considered this fact by applying the JKR model to the spherical punch solution and showed that when the dimensionless parameter,

$$
m=\left(4 R E^{*} / 3 \pi G\right)^{1 / 3},
$$

approaches a value of 10 , the JKR theory with the Hertz approximation is valid.

\section{MATERIALS AND METHODS}

Nanoindentation experiments were performed on RTV615A silicone rubber (GE Silicones, Waterford, NY) mixed with RTV615B curing agent at a 10:1 ratio by weight. The specimen thickness was approximately $1 \mathrm{~mm}$, which was about two orders of magnitude larger than the indentation depth; thus, the JKR assumption of an infinite half space was justified. Experiments were performed using an MFP-3D-BIO AFM (Asylum Research, Santa Barbara, CA). The AFM cantilevers used were made of silicon with a nominal spring stiffness of $0.6 \mathrm{~N} / \mathrm{m}$ with $5-\mu \mathrm{m}$ glass spheres (Novascan Technologies, Ames, IA) attached to their tips. The AFM cantilever stiffness was calibrated to be $0.642 \mathrm{~N} / \mathrm{m}$ using the thermal tune method, ${ }^{9}$ which was built into the MFP-3D software. The nanoindentation experiments with the AFM used the software's force-distance mode. The scanner traveled a distance of $3 \mu \mathrm{m}$, and the maximum cantilever deflection during contact was set to $300 \mathrm{~nm}$ to prevent excessive cantilever bending. Multiple force curves were recorded at scan rates of $0.06,0.60$, and $6.00 \mu \mathrm{m} / \mathrm{s}$. All force curves collected demonstrated a high level of repeatability, suggesting that the material was not rate dependent.

The raw data, given by the cantilever deflection and scanner position, was converted to force and indentation depth by multiplying the cantilever deflection by the spring stiffness and taking the difference of the scanner position and the cantilever deflection. Then the curves were fit to the JKR equations [Eqs. (3)-(5)] with the reduced modulus $E^{*}$ left as an unknown. However, this calculation assumed that there was no indentation upon contact. As discussed previously, this assumption may not be true due to surface forces and long-range attractive forces. To accurately identify the contact point, a leastsquare fitting procedure was used with a shift in the indentation data $h_{0}$ as an unknown. Leaving the value of $h_{0}$ as an unknown in the fitting procedure was the most accurate way of determining this shift. ${ }^{13}$ As previously mentioned, the energy release rate could change during the experiment, so the value of work of adhesion determined from the pulloff force could not be used for energy release rate in the fitting. Therefore, the energy release rate $G$ was also left as an unknown in the fitting. To solve for $E^{*}, h_{0}$, and $G$ simultaneously, a least-square error-minimization algorithm was written in Matlab (MathWorks, Natick, MA), which minimizes the mean-square difference between the load values measured experimentally and calculated using the JKR theory. To test the robustness of the error-minimization algorithm, studies were performed using the minimization algorithm with noise introduced into the data and with changes in initial guesses. It was found that the solutions of the minimization algorithm were unique, indicating the robustness of the algorithm to different experimental conditions.

A displacement-controlled uniaxial tension experiment was conducted to independently verify the value of reduced modulus obtained in the nanoindentation experiments. In the tension experiment, a portion of the PDMS specimen used in nanoindentation was cut to a test size of $18 \mathrm{~mm} \times 3 \mathrm{~mm} \times 1 \mathrm{~mm}$. A M410-2S motorized translation stage (Physik Instrumente, Irvine, CA) deformed the specimen to a true strain of approximately $18 \%$, and a 500-g load cell (LCFA-500g; Omega Engineering, Stamford, CT) measured the load. Horizontal lines were drawn on the specimen, and images of the specimen were captured at strain increments of approximately $2 \%$ with a digital camera with a $12-\mathrm{mm}$ lens. The relative motion between the horizontal lines was used to determine the strain in the specimen to an accuracy of about $0.5 \%$. The results from the uniaxial tension experiment showed that specimens were linear throughout the range of strains tested with a Young's modulus of $0.89 \pm$ $0.04 \mathrm{MPa}$. Poisson's ratio was measured to be $0.47 \pm 0.02$ using the same tension experiment setup with a specimen size of $20 \mathrm{~mm} \times 15 \mathrm{~mm} \times 1 \mathrm{~mm}$. 


\section{RESULTS}

Nanoindentation experiments are performed using an AFM on PDMS. It is found that the data collected at rates of $0.06,0.60$, and $6.00 \mu \mathrm{m} / \mathrm{s}$ are nearly identical, indicating a negligible viscoelastic (rate) effect. The forcedistance results from a loading rate of $0.60 \mu \mathrm{m} / \mathrm{s}$ are shown in Fig. 2 (dotted line). It should be noted that this raw data has not been corrected for the uncertainty in the point of initial contact. The force-indentation depth data from the nanoindentation experiment is fit to the JKR model [Eqs. (3)-(5)] using the error-minimization procedure described earlier. The contact point is established by shifting the raw data in Fig. 2 by some constant $h_{0}$, where $h_{0}$ is a fitting parameter of the error-minimization procedure. The force-indentation data that is shifted by this constant is also plotted in Fig. 2 (solid line). The minimum force in the loading curve of Fig. 2 corresponds to an indentation depth of approximately $0.03 \mu \mathrm{m}$.

A qualitative evaluation of the fit to the JKR model is shown in Fig. 3. The data collected while the AFM scanner is engaging (loading) is plotted with the fit to the data in Fig. 3(a). A comparison between the fit and the data indicates good agreement. During fitting near the maximum load, some deviation between the experimental data and the JKR model is observed, as shown in Fig. 3(a) (inset). This deviation is likely caused by nonlinearity in the cantilever deflection measurement because of large cantilever deformation. When fitting, the in-contact data near the maximum load is cropped to avoid any errors this nonlinearity could cause. The average value of reduced modulus $E^{*}$ obtained by fitting 20 sets of force-indentation curves collected at a rate of $0.60 \mu \mathrm{m} / \mathrm{s}$ is $1.12 \mathrm{MPa}$ with a standard deviation of $0.01 \mathrm{MPa}$. The low standard deviation indicates that the data is highly repeatable, implying

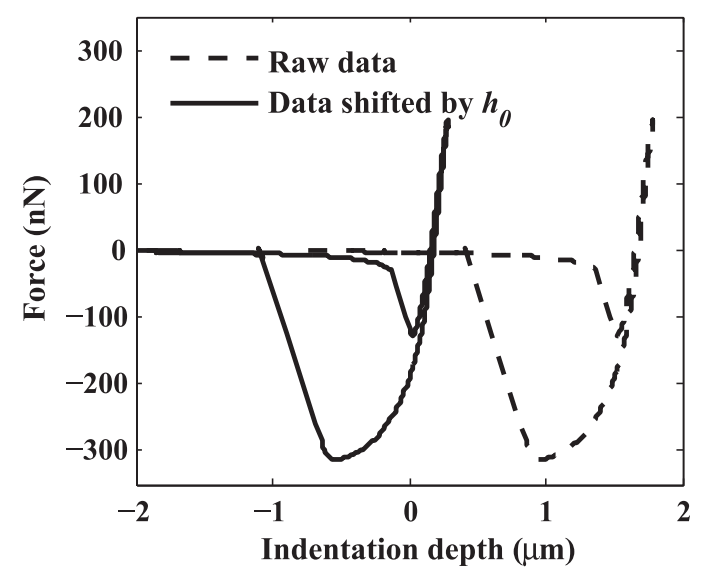

FIG. 2. Plot of force-indentation depth data during nanoindentation experiments on PDMS (dotted line). Because of uncertainty in the contact point, the raw data must be shifted by the value of $h_{0}$ calculated in the JKR fitting (solid line). The minimum force of the loading curve occurs at an indentation depth of $0.03 \mu \mathrm{m}$. Data is collected at a scanner rate of $0.60 \mu \mathrm{m} / \mathrm{s}$. that the substrate deformation remained elastic. The reduced modulus from data collected at slower and faster rates is found to be within $3 \%$ of the modulus found at $0.60 \mu \mathrm{m} / \mathrm{s}$. By using the value of Poisson's ratio measured in the tensile experiments of 0.47 , Young's modulus is calculated to be $0.87 \mathrm{MPa}$, which agrees well with the value of Young's modulus determined using the tensile experiment.

The data collected while the scanner is withdrawing (unloading) is also fit to the JKR model using the value of shift $h_{0}$ and reduced modulus $E^{*}$ found in the fitting to the loading data but a different value of energy release rate $G$ [Fig. 3(b)]. However, as can be seen by observing the poor fit in Fig. 3(b), the experimental data during unloading does not match the JKR model. Especially noteworthy is the fact that the spherical indenter stays in contact with the PDMS specimen for a longer time than the JKR theory predicts. The least-square fitting is repeated by allowing both the reduced modulus and the energy release rate to change, but only minor improvement in the fitting is found. Additionally, the use of the energy release rate computed from the pull-off force using either the load-controlled assumption [Eq. (6)] or the displacement-controlled assumption [Eq. (7)] does not improve the fitting. To further analyze the unloading data, the dimensionless parameter $m$ given in Eq. (8) is calculated to be approximately 5 , which is smaller than the required value of 10 to apply the Hertz approximation of small displacements with the JKR theory. ${ }^{25}$ Based on the analysis in Ref. 25 , the use of the Hertz approximation could lead to errors in computation of the radius of contact of about $1 \%$ at low loads and $10 \%$ at the maximum load. Therefore, the data is fit to the spherical punch solution with the JKR adhesion model incorporated given in Ref. 25. Again, no improvement in the fitting is found. Thus, it appears that the extended period of contact between the indenter and the specimen measured during unloading is not appropriate for fitting to the JKR model.

\section{DISCUSSION}

Previous articles have discussed the importance of accurate determination of the contact point, and they have suggested various methods for introducing a shift in the data to determine the contact point. ${ }^{12-15}$ In this study, after shifting the data by $h_{0}$ by using the least-square errorfitting algorithm, the indentation depth associated with the smallest force during loading is approximately $0.03 \mu \mathrm{m}$. For more adhesive materials, the indentation depth associated with the lowest load could be far larger than the $0.03 \mu \mathrm{m}$ found for PDMS. Although $0.03 \mu \mathrm{m}$ seems small, it has been found here that because of the nonlinear nature of the equations, the resulting value of reduced modulus can be heavily dependent on the shift value $h_{0}$. To illustrate this point, the data is fit to the JKR equations by qualitatively selecting a value of shift to be the measured indentation that 

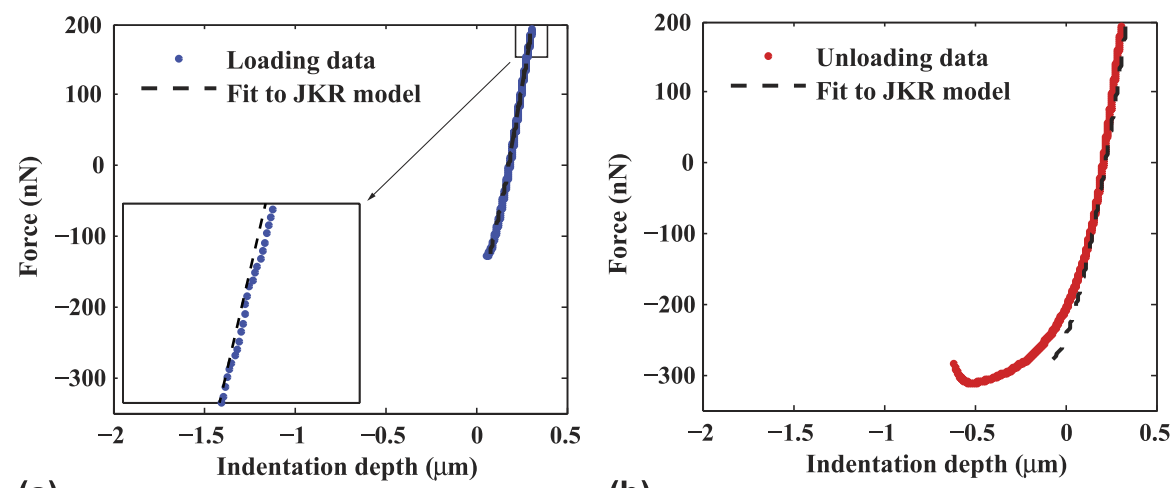

(a)

(b)

FIG. 3. Force-indentation depth data on PDMS collected during the (a) loading and (b) unloading phases. The data is fit to the JKR model, shown by the dotted lines. The inset of (a) demonstrates a nonlinearity in the data at maximum force that must be cropped out when fitting to the JKR model. The data plotted here is down-sampled for clarity. Data is collected at a scanner rate of $0.60 \mu \mathrm{m} / \mathrm{s}$.

corresponds to the smallest force recorded during loading. Such an estimate in the value of $h_{0}$ would appear to be justified on the basis of a qualitative inspection of Fig. 2 (solid line), which shows the contact point for the loading phase is near to the point of minimum force. However, such an estimate leads to a calculated value of reduced modulus of approximately $1.59 \mathrm{MPa}$, which is $42 \%$ larger than the computed value when the shift $h_{0}$ is included as a fitting parameter. Therefore, it is recommended here that $h_{0}$ be a fitting parameter in a nonlinear least-square error-fitting procedure because employing a fitting parameter makes use of as much of the experimental data as possible, which gives the best probability for an accurate calculation of reduced modulus.

If, instead of using an erroneous value of shift $h_{0}$, an incorrect value of energy release rate is chosen, errors are also large. For example, if the value of energy release rate is taken from the work of adhesion calculated using the pulloff force with Eq. (6) and it is used to calculate the reduced modulus, a value of $0.83 \mathrm{MPa}$ is obtained, which is $26 \%$ smaller than the calculated value using the energy release rate as an unknown in the fitting. Therefore, it appears that the value of energy release rate, like the value of shift $h_{0}$, has a significant effect on the fitting. A more quantitative study of the energy release rate can be performed by using the data collected during loading and unloading to calculate the contact radius by using Eq. (3). Then the energy release rate is calculated during loading and unloading by using Eq. (4), and it is plotted in Fig. 4.

An observation from the curves in Fig. 4 is that the average value of energy release rate is different between the data collected during the loading and unloading phases. This hysteresis in energy release rate between loading and unloading has been reported for other systems. ${ }^{19}$ Although this change in energy release rate between loading and unloading is observed for data collected at all three rates, it is slightly smaller for the slower rates than for the faster rates, indicating a slight rate dependence of adhesion. However,

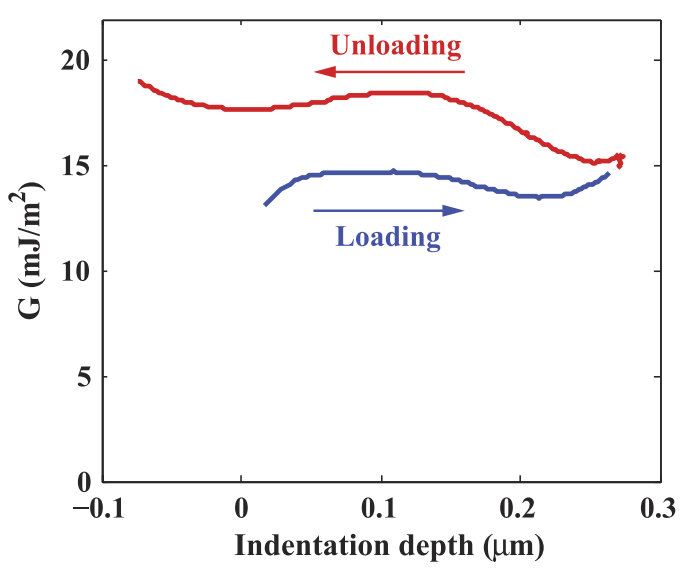

FIG. 4. Energy release rate $G$ computed from data collected in a nanoindentation experiment on PDMS. Data is collected at a scanner rate of $0.60 \mu \mathrm{m} / \mathrm{s}$.

during the individual loading and unloading phases, the change in energy release rate is less than $\pm 10 \%$ from the mean value, indicating that the use of a constant value of energy release rate is reasonable.

Because rate has been shown to affect energy release rate of soft materials,${ }^{20}$ it is possible that a nonconstant rate of growth of the contact radius could have an effect on the energy release rate. Therefore, the contact radius growth rate $d a / d t$ is calculated. In principle, $d a / d t$ could be calculated by differentiating either Eq. (3) or Eq. (5) with respect to time. However, since $G$ is calculated from the measured data for $h$ and $P$, Eq. (5) is likely to be less accurate than Eq. (3). Thus, Eq. (3) is differentiated in time and rearranged,

$$
\frac{d a}{d t}=\left(\frac{2 a}{3 R}-\frac{P}{2 E^{*} a^{2}}\right)^{-1}\left(\frac{d h}{d t}-\frac{1}{2 E^{*} a} \frac{d P}{d t}\right) .
$$

Both $d h / d t$ and $d P / d t$ are calculated numerically, and a plot of $d a / d t$ is shown in Fig. 5. This plot shows that 
indeed the change in contact radius is not constant in time. However, it is noted that there does not appear to be any correlation between energy release rate and rate of change of contact radius with time.

The data in Fig. 5 also shows a very high value of $d a / d t$ at the start of the loading portion of the nanoindentation experiment. This high value indicates a transient regime, wherein a steady state has not yet been reached, violating the quasistatic assumption in the JKR model. Therefore, this transient data should be excluded during the initial fitting of the data. This observation provides a new objective way to determine which data should be used when fitting nanoindentation data to any quasistatic model, including the JKR model. In this paper, all quantities resulting from fitting the loading data to the JKR model used data that excludes this transient regime by removing data where the calculated value of $d a / d t$ is more than $50 \%$ larger than the average value of $d a / d t$ when steady state is achieved.

In the results section, emphasis is placed on the use of the data collected while engaging to accurately calculate the reduced modulus. Traditional nanoindentation experiments use the stiffness during unloading of the nanoindenter tip because of plasticity that can be introduced while loading. Here, it has been found that the nanoindentation experiments are highly repeatable, indicating that deformations of the PDMS are elastic. As a result, there is no reason that the data collected while engaging should not be used for calculation of the reduced modulus. However, this study has shown that when using an AFM, the data collected during unloading should not be used for calculation of reduced modulus because the unloading data does not fit to the force-distance relationship given by the JKR theory. Although rate of change of contact radius with time and changes in energy release rate could cause this poor fitting, Figs. 4 and 5 show that rate of change of contact radius with time and changes in energy release rate do not vary much more during the unloading

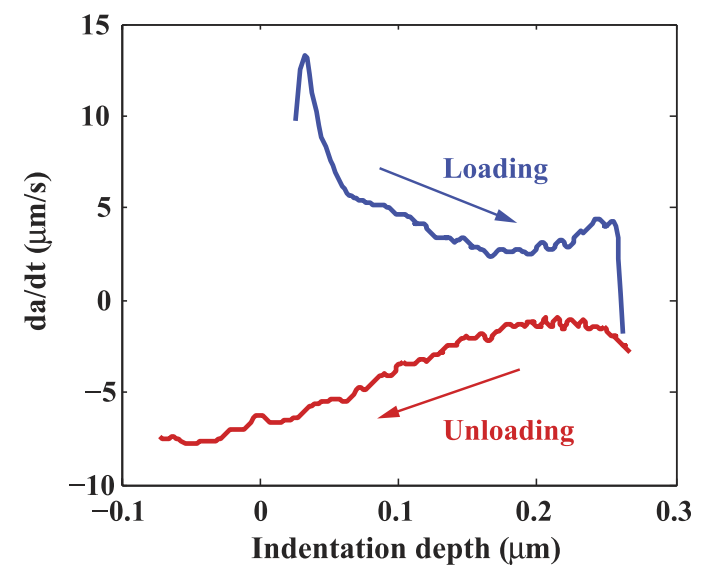

FIG. 5. Rate of change of contact radius $d a / d t$ calculated from data recorded in a nanoindentation experiment on PDMS. Data is collected at a scanner rate of $0.60 \mu \mathrm{m} / \mathrm{s}$. phase than they vary during the loading phase. Therefore, it is unlikely that changes in rate of change of contact radius with time and changes in energy release rate are causing the poor fit to the JKR model.

A more likely cause for the inability to fit the unloading data to the JKR model comes from the compliance introduced into the system through the AFM cantilever. Traditional JKR experiments use the load-controlled model to compute the pull-off force. However, the actual experiment controls the displacement of a flexible cantilever; thus, the experiment is neither load controlled nor displacement controlled. Violation of the assumption of load control creates a more stable system than the JKR equations describe, which increases the amount of time that the tip and specimen are in contact during unloading. This hypothesis of increased stability caused by the stiffness of the system is supported by the data collected during the unloading phase plotted in Fig. 3(b), which shows that the tip and specimen remain in contact for a longer time than the JKR model predicts.

To quantitatively analyze the system stability, consider the reduction in contact radius during the unloading phase as the propagation of a crack with length $c$, where $c$ is given by $c=a_{\max }-a$, with $a_{\max }$ equal to the maximum contact radius. In fracture mechanics, the stability of the system is related to the change in energy release rate with crack length, $d G / d c$. A larger change in energy release rate with crack length indicates a less stable system, and if one assumes that the work of adhesion is constant, the point of instability occurs when the derivative $d G / d c$ becomes positive. By taking into account the stiffness of the cantilever used in this study, $d G / d c$ is calculated (see appendix for details) and plotted in Fig. 6. $d G / d c$ for a load-controlled assumption, corresponding to Eq. (6), is also shown in Fig. 6. As the curves in Fig. 6 show, $d G / d c$ assuming load control is greater than $d G / d c$ computed for the actual experiment. Moreover, under the assumption of load control, the value of $d G / d c$ reaches zero at an indentation depth of approximately $-0.1 \mu \mathrm{m}$, indicating the onset of unstable fracture at the same point that Eq. (6) predicts pull-off from the surface. However, the value of $d G / d c$ calculated from the experimental data is still negative at a depth of $-0.1 \mu \mathrm{m}$, indicating the fracture process is still stable.

One final observation about the data collected during the unloading phase is that the force increases slightly just before pull-off. This behavior is observed for data collected at all three rates and is plotted in Fig. 7 for data collected at a scanner rate of $0.60 \mu \mathrm{m} / \mathrm{s}$. It can be shown that for a system in equilibrium, the derivative of force with respect to indentation depth is related to the system compliance by the equation ${ }^{23}$

$$
\frac{d P}{d h}=-k
$$




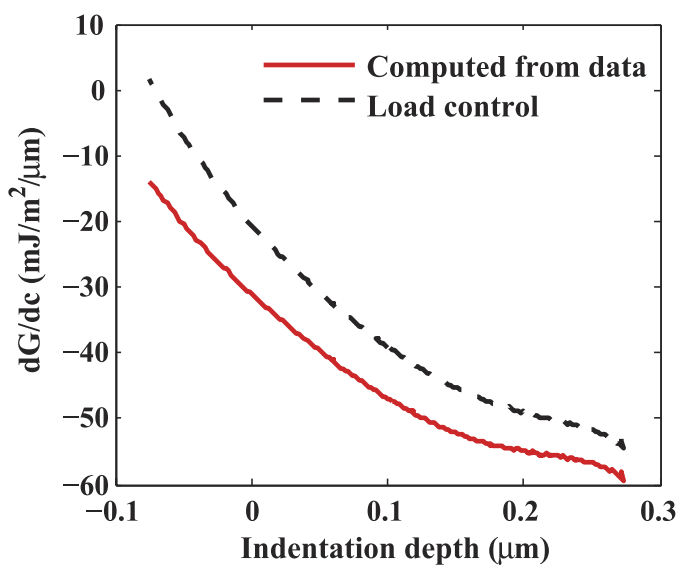

FIG. 6. Rate of change of energy release rate with crack length $d G / d c$ calculated from unloading data from an experiment conducted at a scanner rate of $0.60 \mu \mathrm{m} / \mathrm{s}$ (solid line). The rate of change of energy release rate using a load-controlled assumption is also plotted (dotted line). The fact that $d G / d c$ is greater for the load control case indicates that the assumption of load control is less stable than the actual experiment. Details of the calculations are in the appendix.

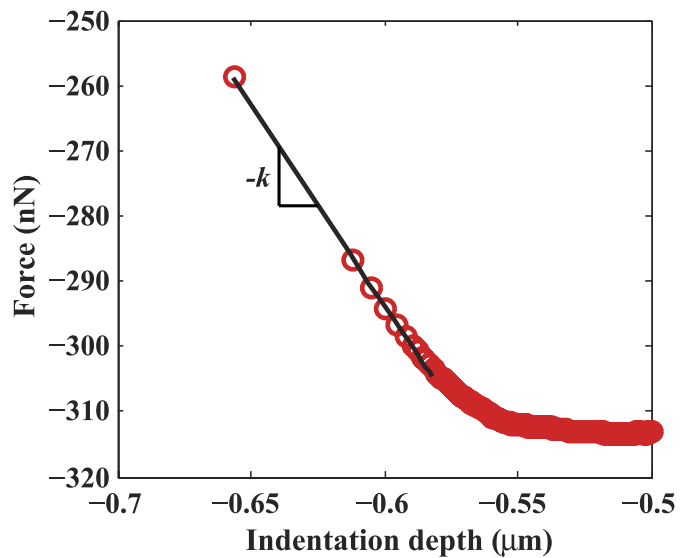

FIG. 7. Detail of data collected at the end of the unloading phase shown in Fig. 3(b). The slope of the line drawn is equal to $-k$, where $k$ is the AFM cantilever spring stiffness.

where $k$ is the stiffness of the system, which in this experiment is equal to the AFM cantilever stiffness. The value of $d P / d h$ calculated from the data collected at all three rates is found to be very repeatable with a mean of $-0.635 \mathrm{~N} / \mathrm{m}$ and a standard deviation of $0.007 \mathrm{~N} / \mathrm{m}$. After applying Eq. (10), the value of $k$ calculated using $d P / d h$ is within $1 \%$ of the value of cantilever stiffness measured using the thermal tune method. The fact that Eq. (10) holds provides further evidence that the stability of the AFM causes a lack of fitting between the JKR model and the experimental data collected during the unloading phase. Furthermore, since Eq. (10) requires that the system remains in equilibrium and it is shown here that Eq. (10) holds for data collected at rates over three orders of magnitude, it can be concluded that the system remains in equilibrium during the indentation experiment.

\section{CONCLUSIONS}

Despite the high force resolution of the AFM, several difficulties-mostly caused by adhesion-prevent the AFM from being more commonly used for nanoindentation of soft and biological materials to obtain reliable reduced modulus measurements. In this paper, a new methodology is provided for the accurate determination of the reduced modulus of soft materials such as PDMS using an error-minimization algorithm with the JKR model by making the following observations:

(i) The calculated value of modulus is heavily dependent on the contact point; therefore, it is necessary to introduce a constant shift in the indentation data. This shift can be determined by fitting the data to the JKR model.

(ii) The mean value of energy release rate changes between the loading and the unloading phases. Thus, the energy release rate cannot be determined from the work of adhesion measured by the pull-off force, and it must be left as a fitting parameter in the JKR model.

(iii) Upon initial contact between the AFM tip and the specimen, there is a transient period wherein the change in radius of contact with time is large. To avoid violating the JKR model's quasistatic assumption, the transient data must be excluded from the JKR fitting.

(iv) It is noted that the data collected during unloading of the AFM cantilever from the specimen does not fit the JKR theory. This observation is caused by the stiffness introduced by the AFM cantilever. To better fit the data collected during the unloading phase, a more compliant AFM cantilever could be used. However, a more compliant cantilever is more likely to deform into a nonlinear regime. Therefore, use of a more compliant cantilever is not recommended here.

(v) Finally, it is demonstrated that the AFM spring stiffness is directly related to the slope of the forceindentation curve at pull-off through Eq. (10). This observation indicates that the system is in equilibrium during the nanoindentation experiments.

\section{ACKNOWLEDGMENTS}

This research was supported by the National Science Foundation (DMR no. 0520565) through the Center for Science and Engineering of Materials (CSEM) at the California Institute of Technology, and this support is gratefully acknowledged. J.N. is supported by the Department of Defense (DoD) through the National Defense Science \& Engineering Graduate Fellowship (NDSEG) Program.

\section{REFERENCES}

1. M. Paszek, N. Zahir, K. Johnson, J. Lakins, G. Rozenberg, A. Gefen, C. Reinhart-King, S. Margulies, M. Dembo, 
D. Boetiger, D. Hammer, and V. Weaver: Tensional homeostasis and the malignant phenotype. Cancer Cell 8, 241 (2005).

2. S. Suresh, J. Spatz, J.P. Mills, A. Micoulet, M. Dao, C.T. Lim, M. Beil, and T. Seufferlein: Connections between single-cell biomechanics and human disease states: gastrointestinal cancer and malaria. Acta Biomater. 1, 15 (2005).

3. D.E. Ingber, L. Dike, L. Hansen, S. Karp, H. Liley, A. Maniotis, H. McNamee, D. Mooney, G. Plopper, J. Sims, and N. Wang: Cellular tensegrity: Exploring how mechanical changes in the cytoskeleton regulate cell growth, migration, and tissue pattern during morphogenesis. Int. Rev. Cytol. 150, 173 (1994).

4. C.M. Lo, H.B. Wang, M. Dembo, and Y.L. Wang: Cell movement is guided by the rigidity of the substrate. Biophys. J. 79, 144 (2000).

5. J.Y. Wong, A. Velasco, P. Rajagopalan, and Q. Pham: Directed movement of vascular smooth muscle cells on gradient-compliant hydrogels. Langmuir 19, 1908 (2003).

6. M. Geerligs, L. van Breemen, G. Peters, P. Ackermans, F. Baaijens, and C. Oomens: In vitro indentation to determine the mechanical properties of epidermis. J. Biomech. 44, 1176 (2011).

7. C. Li, L.A. Pruitt, and K.B. King: Nanoindentation differentiates tissue-scale functional properties of native articular cartilage. J. Biomed. Mater. Res. Part A 78A, 729 (2006).

8. L. Lu, S.J. Oswald, H. Ngu, and F.C-P. Yin: Mechanical properties of actin stress fibers in living cells. Biophys. J. 95, 6060 (2008).

9. J.L. Hutter and J. Bechhoefer: Calibration of atomic force microscope tips. Rev. Sci. Instrum. 64, 1868 (1993).

10. L. Sirghi and F. Rossi: Adhesion and elasticity in nanoscale indentation. Appl. Phys. Lett. 89, 243118 (2006).

11. J.S. Villarrubia: Algorithms for scanned probe microscope image simulation, surface reconstruction, and tip estimation. J. Res. Nat. Inst. Stand. Technol. 102, 425 (1997).

12. S.L. Crick and F.C-P. Yin: Assessing micromechanical properties of cells with atomic force microscopy: Importance of the contact point. Biomech. Model. Mechanobiol. 6, 199 (2007).

13. Y. Cao, D. Yang, and W. Soboyejo: Nanoindentation method for determining the initial contact and adhesion characteristics of soft polydimethylsiloxane. J. Mater. Res. 20, 2004 (2005).

\section{APPENDIX}

As mentioned in the discussion section, the derivative $d G / d c$ is related to the stability of the system. Here, $d G / d c$ is calculated by taking into account the compliance introduced by the AFM cantilever. It can be shown ${ }^{26}$ that for a system with finite compliance,

$$
\frac{d G}{d c}=\left(\frac{\partial G}{\partial c}\right)_{P}-\left(\frac{\partial G}{\partial P}\right)_{c}\left(\frac{\partial h}{\partial c}\right)_{P}\left[C_{m}+\left(\frac{\partial h}{d P}\right)_{c}\right]^{-1},
$$

where $c$ is the crack or debond length, $C_{m}$ is the compliance introduced by the AFM cantilever, $C_{m}=1 / k$, and $k$ is the cantilever's spring constant. These derivatives can be calculated from the equations given by Maugis $^{23}$ :

$$
\left(\frac{\partial G}{\partial A}\right)_{P}=\frac{3\left(P_{1}^{2}-P^{2}\right)}{16 a^{5} E^{*}}
$$

14. D.C. Lin, E.K. Dimitriadis, and F. Horkay: Robust strategies for automated AFM force curve analysis-I. Non-adhesive indentation of soft, inhomogeneous materials. J. Biomech. Eng., 129, 430 (2007).

15. J.D. Kaufman and C.M. Klapperich: Surface detection errors cause overestimation of the modulus in nanoindentation on soft materials. J. Mech. Behav. Biomed. Mater. 2, 312 (2009).

16. A.J. Moseson, S. Basu, and M.W. Barsoum: Determination of the effective zero point of contact for spherical nanoindentation. J. Mater. Res. 23, 204 (2008).

17. S.R. Kalidindi and S. Pathak: Determination of the effective zeropoint and the extraction of spherical nanoindentation stress-strain curves. Acta Mater. 56, 3523 (2008).

18. P. Silberzan, S. Perutz, E.J. Kramer, and M.K. Chaudhury: Study of the self-adhesion hysteresis of a siloxane elastomer using the JKR method. Langmuir. 10, 2466 (1994).

19. K.R. Shull: Contact mechanics and the adhesion of soft solids. Mater. Sci. Eng., R 36, 1 (2002).

20. M.A. Meitl, Z-T. Zhu, V. Kumar, K.J. Lee, X. Feng, Y.Y. Huang, I. Adesida, R.G. Nuzzo, and J.A. Rogers: Transfer printing by kinetic control of adhesion to an elastomeric stamp. Nat. Mater. 5, 33 (2006).

21. K.L. Johnson, K. Kendall, and A.D. Roberts: Surface energy and contact of elastic solids. Proc London, Ser. A. Adv. Math. Phys. 324, 301 (1971).

22. H. Hertz: Üeber die berührung fester elastischer körper. J. Reine Angew. Math. 92, 156 (1881).

23. D. Maugis: Contact, Adhesion and Rupture of Elastic Solids (Springer, Berlin, Germany, 2000), p. 141, 264-272.

24. I.A. Sneddon: The relation between load and penetration in the axisymmetric Boussinesq problem for a punch of arbitrary profile. Int. J. Eng. Sci. 3, 47 (1965).

25. D. Maugis: Extension of the Johnson-Kendall-Roberts theory of the elastic contact of spheres to large contact radii. Langmuir. 11, 679 (1995).

26. T.L. Anderson: Fracture Mechanics: Fundamentals and Applications, 3rd ed. (Taylor \& Francis Group, Boca Raton, FL, 2005), pp. 91-92.

$$
\left(\frac{\partial G}{\partial P}\right)_{c}=\frac{P-P_{1}}{4 \pi a^{3} E^{*}}
$$

where $P_{1}=4 a^{3} E^{*} / 3 R$. By the chain rule,

$$
\left(\frac{\partial G}{\partial A}\right)_{P}=\left(\frac{\partial G}{\partial c}\right)_{P}\left(\frac{\partial c}{\partial a}\right)_{P}\left(\frac{\partial a}{\partial A}\right)_{P},
$$

where $(\partial c / \partial a)_{P}=-1$ and $(\partial a / \partial A)_{P}=(2 \pi a)^{-1}$. Finally, the derivatives of indentation depth are calculated,

$$
\left(\frac{\partial h}{\partial c}\right)_{P}=\left(\frac{\partial h}{\partial a}\right)_{P}\left(\frac{\partial c}{\partial a}\right)_{P}=-\left(\frac{\partial h}{\partial a}\right)_{P}=-\frac{2 a}{3 R}+\frac{P}{2 a^{2} E^{*}} \quad,
$$

$$
\left(\frac{\partial h}{d P}\right)_{c}=\frac{1}{2 a E^{*}}
$$


Combining the above equations together gives the equation for $d G / d c$ for the system. To calculate $d G / d c$ using the JKR model, one assumes a loadcontrolled system. The stability equation for load control is given by

$$
\left(\frac{\partial G}{\partial c}\right)_{J K R}=\left(\frac{\partial G}{\partial c}\right)_{P}
$$

which can be calculated from Eq. (A4) above. 


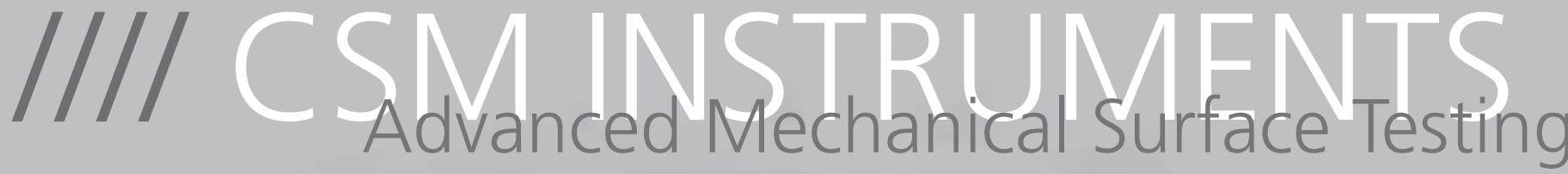

\section{INSTRUMENTED NANOINDENTATION, SCRATCH AND TRIBOLOGY}

\section{//// CSM Instruments ULTRA NANOINDENTATION TESTER (UNHT) The new generation of Nanoindenter with ultra low thermal drift}

This new Nanoindenter is based on the principle of using a measurement head (built with drift-free Zerodur $^{\odot}$ glass material) with one axis for measurement and one axis for reference, each one having its own actuator and its own sensors of depth and force. It is therefore possible to carry out active referencing of the surface of the sample. The true penetration depth is measured with a third capacitive sensor monitoring the direct difference of penetration between the reference and the indenter.
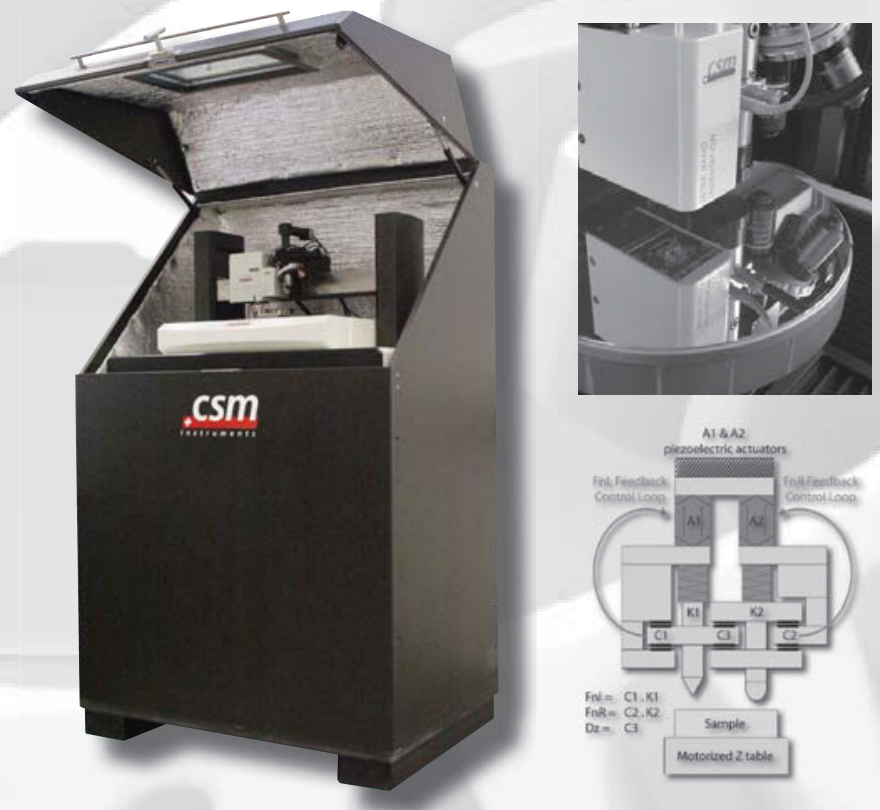

\section{$>$ Ultra low load indentations on low-K films}

Low load indentations on low-K films of $200 \mathrm{~nm}$ thickness demonstrate the high-resolution capabilities of this unique and patented UNHT instrument. This makes it the ideal instrument for characterization of ultra thin coatings.

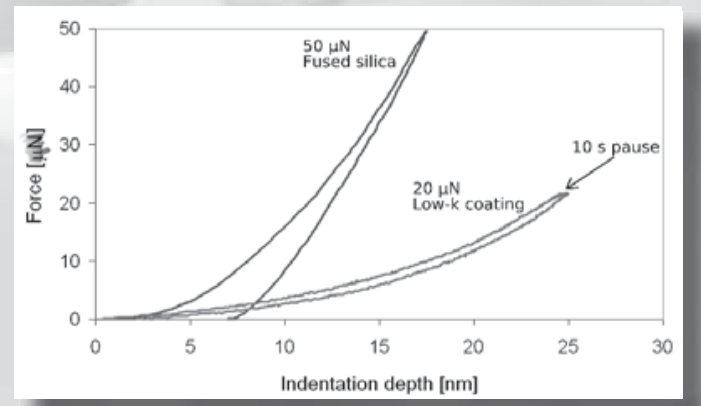

\section{$>$ Extremely soft materials: gels and cells}

Experiments performed on gels show the exceptional capabilities of the UNHT in extreme applications: with loads lower than $50 \mu \mathrm{N}$, penetration depths can reach 10 to $20 \mu \mathrm{m}$ ! Great measurement accuracy can be obtained for penetration depths from $10 \mathrm{~nm}$ to over $50,000 \mathrm{~nm}$. Thanks to the unmatched thermal stability of the system, adhesion energy can be easily studied on various soft materials.

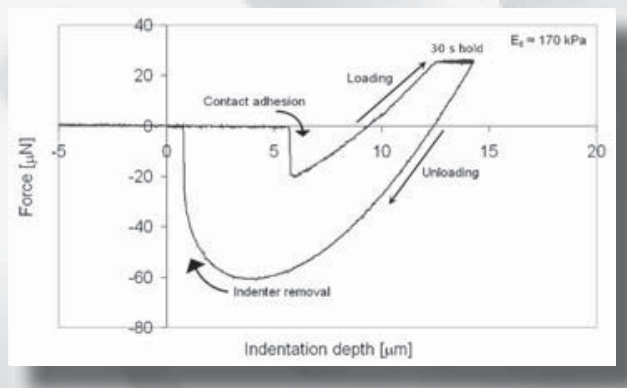

\section{$>$ Creep studies on polymers}

Nanomechanical testing of materials exhibiting timedependent properties requires an instrument with high thermal stability and high resolution. Creep studies on polymers can always be performed without any thermal stabilization period or thermal drift correction!

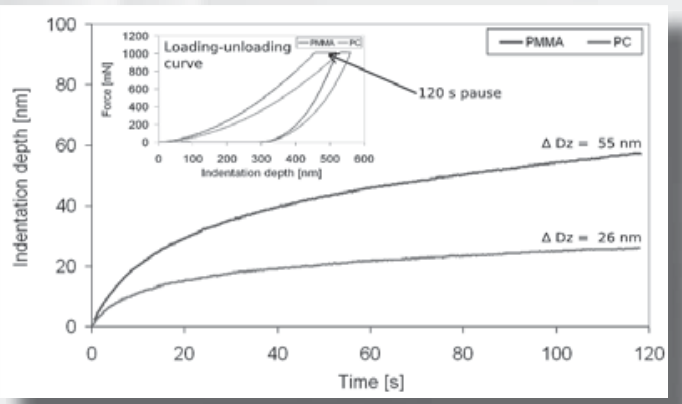

